\title{
The Impact of Olfactory Dysfunction on the Psychological Status and Quality of Life of Patients with Nasal Obstruction and Septal Deviation
}

\author{
Konstantinos Valsamidis ${ }^{1}$ Athanasia Printza ${ }^{1}$ Jannis Constantinidis ${ }^{1}$ Stefanos Triaridis ${ }^{1}$ \\ ${ }^{1} 1$ st Otolaryngology Department, Medical school, Aristotle \\ University of Thessaloniki, AHEPA Hospital, 54124, Thessaloniki, \\ Greece \\ Address for correspondence Stefanos Triaridis MD, MsC, PhD, 1st \\ Otolaryngology Department, Medical school, Aristotle University of \\ Thessaloniki, AHEPA Hospital, 54124, Thessaloniki, Greece \\ (e-mail: triaridis@hotmail.com).
}

Int Arch Otorhinolaryngol 2020;24(2):e237-e246.

\begin{abstract}
Introduction Olfactory dysfunction may be present in patients with nasal obstruction and septal deviation. The impact of olfactory dysfunction on the psychological profile and quality of life (QoL) of these patients remains unexplored.

Objective The present study aimed to investigate the emotional status and QoL of patients with olfactory dysfunction and septal deviation and to identify predictors associated with clinically significant improvement of psychological status and QoL, focusing mainly on the role of olfactory recovery after septoplasty.

Methods The olfactory function was quantitatively assessed using the "Sniffin" sticks" test (Burghart Messtechnik GmbH, Wedel, Germany) in 60 patients and 25 controls enrolled in this prospective study. The participants completed validated questionnaires specific for general health (Short Form-36), nasal-symptom related QoL (SinoNasal Outcome Test-22), olfaction-associated QoL (Questionnaire of Olfactory Deficits) and for assessing their psychological state (Short Anxiety Screening Test and Beck Depression Inventory) preoperatively and 6 months postoperatively. The patients used the Glasgow Benefit Inventory to evaluate their personal benefit after septoplasty with.

Results Septoplasty led to significantly improved olfactory function. Patients with olfactory impairment had significantly lower nasal-symptom related QoL, higher stress levels, and more depressive mood compared with normosmics and controls before and

\section{Keywords}

- olfactory dysfunction

- septoplasty

- anxiety

- emotional status

- quality of life

- olfaction test after septoplasty. Postoperatively, personal benefit from surgery was higher in normosmic patients. Improvement of nasal-symptom related QoL was significantly associated with higher likelihood of clinically significant improvement of patients' psychological profile and more personal benefit from surgery. Olfactory dysfunction was negatively correlated with the emotional status of the patients.

Conclusion Olfactory dysfunction appears to significantly affect the psychological status of patients with nasal obstruction, and olfactory recovery improves the patients' perception of personal benefit from septoplasty.
\end{abstract}

received

August 5, 2019

accepted

December 3, 2019

published online

February 27, 2020
DOI https://doi.org/

10.1055/s-0040-1701269.

ISSN $1809-9777$.
Copyright $(2020$ by Thieme Revinter

Publicações Ltda, Rio de Janeiro, Brazil
License terms

(요 $\Theta \circledast$ 


\section{Introduction}

Olfactory dysfunction due to decreased nasal airflow is a common symptom among patients with nasal obstruction related to nasal septal deviation. ${ }^{1}$ Olfactory disorders may have a significant negative impact on different areas of daily life, including food appreciation, safety (detection of environmental hazards, such as smoke, fire, gas, and spoiled food), personal hygiene, and social communication. Especially in the case of individuals whose professions depend on a well-functioning sense of smell, such as cooks, wine tasters, perfumers, or firemen, olfactory deficits can be catastrophic. $^{2}$ Therefore, olfactory impairment due to septal deviation, although underestimated by patients and overlooked by doctors, ${ }^{3}$ may substantially affect the quality of life (QoL) of patients and potentially be associated to mental health. ${ }^{2,4-6}$ Nasal septoplasty is the standard treatment of nasal septal deviation because it usually produces an improvement of nasal airflow and resolution of nasal obstruction symptoms. ${ }^{7-10}$ To date, there are a few studies evaluating the effects of septal surgery on olfactory function. ${ }^{1,11-18}$ However, the impact of olfactory impairment due to septal deviation on the nasal-symptom related and general QoL of the patients has not been adequately addressed in the literature. In addition, although there are studies investigating predictive factors for QoL outcomes after septoplasty, ${ }^{19-25}$ there is no data available to the relevance of the olfactory function specifically to patients' psychological status (anxiety and depression symptoms) and its effect on their satisfaction with the surgical procedure and postoperative QoL recovery. The use of standardized olfactory tests, validated olfaction-specific questionnaires, and mental health psychometric instruments allows a clearer delineation of the psychological profile and evaluation of olfaction of the patients as a codeterminant for QoL and emotional status after septoplasty. The clinical importance is that these data may enable otorhinolaryngologists to better assess their patients and inform them about the anticipated benefit for their QoL after septoplasty.

The aim of the present prospective study was to assess the emotional status and QoL outcomes of patients with olfactory dysfunction and nasal obstruction symptoms who undergo septoplasty. Additionally, the study explored differences in QoL and psychological status between normosmic patients and those with olfactory deficits and investigated the prognostic value of demographic and clinical characteristics for clinically significant improvement of QoL, anxiety symptoms, and emotional status of patients with olfactory dysfunction.

\section{Materials and Methods}

This was a prospective observational study. Sixty adult patients with nasal obstruction due to nasal septal deviation were studied. The diagnosis of septal deviation was established based on clinical examination and nasal endoscopy. Twentyfive healthy individuals, who had neither nasal obstruction nor septal deviation, were recruited as controls. All the enrolled participants signed the informed consent. The study protocol was performed according to the Declaration of Helsinki and was approved by the local institutional review board (decision no 3525/10.02.2016).

Adult patients younger than 65 years with nasal septal deviation, diagnosed by means of clinical examination and presence of symptoms of nasal obstruction for at least 6 months, were included in the study. Patients were excluded if they met any of the exclusion criteria, such as chronic rhinosinusitis, with or without nasal polyposis; allergic rhinitis; previous nasal surgical procedure; sinonasal malignancy; perforation of the nasal septum; nasal valve collapse; craniofacial syndrome; fracture or trauma of the nose during the last 3 months; pregnancy or cognitive impairment. Patients with a recent infection of the upper respiratory tract, a known hyposmia, patients exposed to chemicals or those suffering from any neurological or systematic diseases with potential impact on the olfactory function were not included in the study. Additionally, patients who underwent any other nasal surgical procedure, particularly rhinoplasty and sinus surgery concomitant to septoplasty, were also excluded.

All participants filled in a detailed health form, which included demographic information and presence of medical comorbidities, and focused on factors that might affect olfactory function, such as smoking habits, profession, medical treatment, and recreational activities. For recruitment to the study, in addition to anterior rhinoscopy and nasal endoscopy, participants underwent skin prick testing for atopy and sinus computed tomography scanning (when medically justified) for the identification of those who met the exclusion criteria.

The “Sniffin' sticks" test package (Burghart Messtechnik GmbH, Wedel, Germany), which includes specific tests for odor threshold (OT), odor discrimination (OD), and odor identification $(\mathrm{OI})^{26-28}$ was used for quantitative evaluation of the olfactory function of all participants. The results of each test were combined with an overall "Threshold Discrimination Identification (TDI) score"28,29. The TDI score ranges from 0 to 48 . Values of 16 or less represent anosmia; values between 16.25 and 30.5 represent hyposmia; and values over 30.75 represent normosmia. ${ }^{28} \mathrm{~A}$ six-point difference in the TDI score was considered a clinically significant change of olfactory function after surgery. ${ }^{30}$

The olfactory tests were performed preoperatively and 6 months after septoplasty. At these time points, the participants also filled in six widely used questionnaires, translated, and validated into the Greek language: the Nasal Obstruction Symptom Evaluation (NOSE) questionnaire, assessing the severity of nasal obstruction symptoms; ${ }^{31,32}$ the SinoNasal Outcome Test-22 (SNOT-22), assessing nasal symptom-related QoL; $;^{33,34}$ the Questionnaire of Olfactory Deficits (QOD), assessing olfaction-associated QoL; ${ }^{35,36}$ the Short Anxiety Screening Test (SAST), evaluating anxiety symptoms; ${ }^{37,38}$ the Beck Depression Inventory (BDI), evaluating the emotional status of the patients; ${ }^{39,40}$ and the Short Form 36 (SF-36), assessing general QoL. ${ }^{41,42}$ A postoperative general-health survey specific for patients' satisfaction with the surgical procedure, the Glasgow Benefit Inventory (GBI), ${ }^{43,44}$ was completed at 6 months after septoplasty. The NOSE is a 
questionnaire for the evaluation of nasal obstruction. It consists of five questions that are scored on a five-point Likert scale from zero to four. The final score ranges between 0 (no symptoms) and 100 (severe nasal obstruction). The SNOT-22 is a health-related questionnaire, in which patients grade 22 different symptoms related to both nasal and general health, physical and emotional status on a scale from 0 (no symptoms) to 5 (symptoms as severe as can be). The QOD is an olfactionspecific QoL questionnaire, which evaluates the effect of olfactory dysfunction on several areas of daily life. Patients answer to 254 -point scale statements (17 "negative", 2 "positive", 6 "socially desired"). The maximum total score is 57 , and high scores represent a high negative impact of olfactory disorders on QoL. The SAST consists of 10 questions related to somatic symptoms manifestations of anxiety. Each item is self-rated from 1 to 4 , and the total score ranges between 10 and 40, with higher scores indicating a higher degree of anxiety. The BDI is a screening instrument for the evaluation of the psychological profile. It is a 21-item self-reporting questionnaire, and each item is graded from 0 to 3. A higher score is associated with higher levels of depressive mood. The SF-36 is an overall-health related survey assessing QoL in eight domains covering aspects from both physical and mental health from the perspective of patients suffering from chronic diseases. Scores range from 0 to 100 , with a higher score indicating better general QoL. Finally, the GBI is a validated 18 -item postintervention questionnaire measuring the patient's benefit, developed especially for surgical interventions. The total score ranges from +100 (maximum positive change) to -100 (maximum negative change).

All patients underwent surgery under general anesthesia. The same consultant surgeon performed all the operations and did not participate in the questionnaires' collection and analysis of data. The standardized surgical procedure included partial resections and reshaping of the deviated areas of the septum and submucosal radiofrequency tissue ablation for volume reduction of the inferior nasal turbinates. The anterior nasal packings were removed 48 hours after surgery, and the silicone septal splints were removed on the $4^{\text {th }}$ postoperative day. No major complication was encountered postoperatively.

\section{Statistical Analysis}

The data were analyzed with IBM SPSS Statistics for Windows version 25.0 (IBM Corp., Armonk, NY, USA). Descriptive statistics were obtained; quantitative variables are expressed as means with standard deviation (SD) while qualitative variables are presented as frequencies (percentages). The normality of the variables was ascertained with the Kolmogorov-Smirnov test. Differences between not normally distributed data were assessed with the use of the Mann-Whitney $U$ test and Wilcoxon signed-rank test for independent and related samples respectively. For differences in qualitative parameters between groups, the Chi-square test was applied. Univariate linear regression analysis was used for the evaluation of any potential association between the likelihood of clinically significant improvement for each QoL questionnaire and patients' demographic and clinical characteristics. Clinically significant improvement for each QoL questionnaire was defined as a change of $\geq 1 / 2$ SD of the preoperative score. ${ }^{45}$ Multivariate stepwise logistic regression analysis was performed to explore which of the studied patients' characteristics were independently associated with clinically significant improvement of each QoL questionnaire. Only variables with statistical significance during the univariate regression analysis were incorporated into the multiple regression analysis. Odds ratios (ORs) and 95\% confidence intervals (CIs) were estimated as the measure of association between clinically significant improvement for each QoL questionnaire and all potential predictive factors. A $p$-value of less than 0.05 was considered as the statistical significance level.

\section{Results}

The study cohort consisted of 60 patients (34 [56\%] males - 26 [44\%] females, mean age: $32.98 \pm 11.98$ years) and 25 healthy control subjects ( 13 [52\%] males - 12 [48\%] females, mean age: $29 \pm 8.87$ years). In the patients' subgroup, 20 participants were smokers (33.33\%). Regarding the socioeconomic status, $14(23 \%)$ had low, 24 (40\%) had medium, and 22 (37\%) had high socioeconomic status. In the controls' subgroup, 10 participants were smokers (40\%). Regarding the socioeconomic status, 6 (25\%) had low, 9 (35\%) had medium, and 10 (40\%) had high socioeconomic status. There were no statistically significant differences related to age ( $p=0.151$ - Mann-Whitney $U$ test), gender $(p=0.176)$, smoking habits ( $p=0.143)$, or socio-economic status ( $p=0.942$ - Chi-squared test for the 3 qualitative parameters) between the 2 groups.

Regarding the preoperative quantitative evaluation of the participants' olfactory function with the use of Sniffin' Sticks test, patients presented statistically significant lower olfactory scores (OT, OD, OI, TDI) compared with controls (MannWhitney $U$ test, $p<0.001$ for all the variables). The olfactory scores of patients and controls are presented in -Table 1. Six months after surgery, there was a statistically significant improvement in all olfactory scores (Wilcoxon signed-rank test, $p<0.001$ for OT, OI, TDI, and $p=0.008$ for OD). However, the OT, OD, and TDI scores remained statistically significantly lower compared with the scores of the control group (MannWhitney $U$ test, $p<0.05$ for all parameters). Patients, as expected, had preoperatively statistically significant more severe nasal obstruction symptoms (NOSE scores). The patients' NOSE scores were significantly improved postoperatively but remained lower than the controls' scores.

Regarding QoL and psychological status assessment, there were no statistically significant differences both before and 6 months after surgery in olfaction-associated QoL (QOD scores), emotional status (BDI scores,) and general health status (SF-36 scores-only preoperative evaluation) between patients and controls (Mann-Whitney $U$ test, $p>0.05$ for all parameters). The mean values are presented in - Table 1. Preoperatively, the differences in SF-36 scores between patients and controls were minor; therefore, the questionnaire was not filled in by the participants 6 months after surgery. Patients had preoperatively statistically significantly worse nasal symptom-related QoL (SNOT-22 scores) and higher stress levels (SAST scores) than controls (Mann-Whitney $U$ test, $p<0.001$ 
Table 1 Olfactory function, questionnaires of quality of life and psychological status of the controls and the patient group (preoperatively and 6 months after surgery)

\begin{tabular}{|c|c|c|c|c|}
\hline & \multirow[t]{2}{*}{$\begin{array}{l}\text { Control } \\
(n=25)\end{array}$} & \multicolumn{2}{|l|}{$\begin{array}{l}\text { Patient group } \\
(n=60)\end{array}$} & \multirow[t]{2}{*}{$p$-value } \\
\hline & & preop & postop & \\
\hline \multicolumn{5}{|c|}{ Olfactory scores } \\
\hline OT & $9.2(2.19)$ & $4.78(2.88)$ & $6.98(3.03)$ & $<0.001$ \\
\hline OD & $13.12(1.67)$ & $9(2.76)$ & $11.35(2.45)$ & 0.008 \\
\hline Ol & $13.32(2.18)$ & 10.08 & $12.08(1.97)$ & $<0.001$ \\
\hline TDI & $35.64(2.62)$ & $23.87(4.28)$ & $29.42(4.72)$ & $<0.001$ \\
\hline \multicolumn{5}{|l|}{ QoL tools } \\
\hline SF-36 & $83.48(9.74)$ & $80.20(15.45)$ & & \\
\hline SNOT 22 & $12.9(8.86)$ & $42.93(19.57)$ & $26.7(18.9)$ & $<0.001$ \\
\hline QOD & $6.92(2.08)$ & $8.85(5.11)$ & $7.91(3.42)$ & 0.25 \\
\hline \multicolumn{5}{|c|}{ Psychological tools } \\
\hline SAST & $11.7(6.39)$ & $22.63(7.94)$ & $17.5(4.4)$ & 0.05 \\
\hline BDI & $6.85(3.43)$ & $7.53(5.76)$ & $7(5.36)$ & 0.08 \\
\hline
\end{tabular}

Abbreviations: BDI, Beck depression inventory; OD, olfactory discrimination; OI, olfactory identification; OT, olfactory threshold; QOD, questionnaire of olfactory disorders; QoL, quality of life; SAST, short anxiety screening test; SF-36, short form - 36; SNOT 22, SinoNasal outcome test 22; TDI, threshold discrimination identification.

Bold: Statistically significant difference compared with the control group, $p$-values: pre and posttreatment scores comparisons for the patient group -Wilcoxon Signed-Rank test for paired samples.

Data are expressed as mean values (standard deviation), preop: preoperatively, postop: postoperatively.

for all parameters). Although these parameters were significantly improved postoperatively, they maintained their statistically significant difference between the patients and control groups (-Table $\mathbf{1}$ ).

We further investigated QoL and psychological implications in the subgroup of patients with olfactory dysfunction (according to TDI scores). The preoperative results are presented in - Table $\mathbf{2}$ and the postoperative ones in - Table 3. Preoperatively, patients with decreased olfactory function had worse nasal-symptom related and olfaction-associated QoL, higher stress levels, more depressive mood, and reduced general QoL than normosmic patients. However, only the differences in SNOT-22 and BDI scores reached statistical significance $(p<0.05$ for both). Six months after surgery, normosmic patients had statistically significantly better nasal-symptom related QoL, lower stress levels, better emotional status and more personal benefit from the surgical procedure (GBI scores) than patients who reported olfactory deficits $(p<0.05$ for all parameters). Except for general QoL (SF-36 scores), both patients' groups (normosmics and patients with decreased olfactory status) had worse scores in all the above parameters preoperatively compared with controls. These differences were statistically significant for all scores between the patients with olfactory impairment and healthy controls. For normosmic patients, only differences in SNOT-22 and SAST scores reached statistical significance (Mann-Whitney $U$ test, $p<0.001$ for both). Postoperatively, patients with decreased olfactory function had statistically significantly worse SNOT-22, SAST, and

Table 2 Nasal symptom related quality of life (QoL), olfaction related QoL, stress levels, depressive mood and general health status of controls, patients with olfactory deficits and normosmic patients, preoperatively

\begin{tabular}{|l|l|l|l|l|}
\hline & Controls $(\boldsymbol{n}=\mathbf{2 5})$ & Patients with olfactory deficits $(\boldsymbol{n}=\mathbf{5 0})$ & Normosmic Patients $(\boldsymbol{n}=\mathbf{1 0})$ & $\boldsymbol{p}$-value \\
\hline SNOT 22 & $12.9(8.86)$ & $57.7(14.75)$ & $38.8(9.16)$ & 0.019 \\
\hline QOD & $6.92(2.08)$ & $10.94(2.85)$ & $8.9(2.75)$ & 0.109 \\
\hline SAST & $11.7(6.39)$ & $23.62(7.91)$ & $20.4(7.86)$ & 0.222 \\
\hline BDI & $4.65(2.08)$ & $8.26(2.72)$ & $4.7(2.7)$ & 0.029 \\
\hline SF 36 & $83.48(9.74)$ & $77.65(13.34)$ & $81.2(10.98)$ & 0.086 \\
\hline
\end{tabular}

Abbreviations: BDI, Beck depression inventory; QOD, questionnaire of olfactory disorders; QoL, Quality of life; SAST, short anxiety screening test; SF36, Short Form - 36; SNOT 22, SinoNasal outcome test 22.

Bold: Statistically significant difference compared with control group, $\mathrm{p}$ value: comparisons between normosmics and patients with olfactory dysfunction, all groups comparisons: Mann-Whitney $U$ test for independent samples.

Data are expressed as mean values (standard deviation). n: number of patients. 
Table 3 Nasal symptom-related quality of life (QoL), olfaction-related QoL, stress levels, depressive mood and patient's satisfaction with the surgical procedure of patients with olfactory deficits and normosmic patients, 6 months after surgery

\begin{tabular}{|l|l|l|l|l|}
\hline & Controls $(\boldsymbol{n}=\mathbf{2 5})$ & Patients with olfactory deficits $(\boldsymbol{n}=\mathbf{4 1})$ & Normosmic patients $(\boldsymbol{n}=\mathbf{1 9})$ & $\boldsymbol{p}$-value \\
\hline SNOT 22 & $12.9(8.86)$ & $\mathbf{3 0 . 9 7 ( 1 2 . 1 1 )}$ & $\mathbf{2 2 ( 8 . 7 4 )}$ & 0.014 \\
\hline QOD & $6.92(2.08)$ & $8.27(4.33)$ & $7.02(3.4)$ & 0.14 \\
\hline SAST & $11.7(6.39)$ & $\mathbf{2 0 . 5 8 ( 5 . 6 1 )}$ & $15.57(5.76)$ & 0.045 \\
\hline BDI & $4.65(2.08)$ & $8.09(2.49)$ & $4.63(0.61)$ & 0.028 \\
\hline GBI & & $5.35(2.18)$ & $18.05(3.51)$ & 0.002 \\
\hline
\end{tabular}

Abbreviations: BDI, Beck depression inventory; GBI, Glasgow benefit inventory; QOD, questionnaire of olfactory disorders; QoL, quality of life; SAST, short anxiety screening test; SNOT 22: SinoNasal outcome test 22.

Bold: Statistically significant difference compared with control group, $p$-value: comparisons between normosmic patients and patients with olfactory dysfunction, all groups comparisons: Mann-Whitney $U$ test for independent samples.

Data are expressed as mean values (standard deviation). n: number of patients.

BDI scores than controls (Mann-Whitney $U$ test, $p<0.05$ for all). Normosmic patients showed, postoperatively, statistically significant differences from controls only in SNOT-22 scores (Mann-Whitney $U$ test, $p<0.05$ ).

Among the patients' cohort, clinically significant improvement was observed in 22 patients (36.67\%) for olfactionassociated QoL (QOD score), 32 (53.3\%) for stress levels (SAST scores) and 18 (30\%) for emotional health (BDI scores), while 32 patients (53.3\%) reported a positive change of their general health status (GBI scores) after surgical procedure ( - Tables 4 and $\mathbf{5}$ ). The incidence of clinically significant improvement of the QOD, SAST, BDI scores, and positive change of GBI scores in relation to the patients' demographics and disease characteristics was further analyzed ( - Tables $\mathbf{4}$ and $\mathbf{5}$ ). In the univariate linear regression analysis ( - Table 4 ), it was found that the likelihood of clinically significant improvement of the QOD score was higher for patients with clinically significant improvement of the NOSE and SNOT-22 scores and postoperative normal olfactory function ( $p<0.05$ for all parameters). The incidence of positive change of GBI score was higher for patients with clinically significant improvement of the NOSE and SNOT22 scores 6 months after surgery $(p \leq 0.001-\rightarrow$ Table 4$)$. The likelihood of clinically significant improvement of the SAST score was higher for those patients who had clinically significant improvement of the NOSE and SNOT-22 scores $(p<0.05$

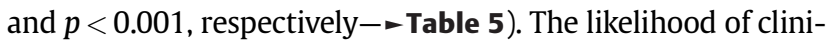
cally significant improvement of the BDI score was higher for patients with clinically significant improvement of the NOSE and SNOT-22 scores and postoperative normal olfactory function $(\boldsymbol{p}<0.05$ for all parameters $-\boldsymbol{-}$ Table $\mathbf{5})$.

The multivariate logistic regression analysis (-Table 6 ) revealed that the clinically significant improvement of the SNOT-22 score was an independent predictive factor significantly associated with higher likelihood of clinically significant improvement of all parameters (QOD score-OR: 3.79, 95\%CI: 1.11-12.88, $p<0.05$; SAST score - OR: 6.14, 95\%CI: 1.87-20.07, $p<0.05$; BDI score-OR: $5.61,95 \% \mathrm{CI}: 1.35-23.15, p<0.05)$ and higher incidence of positive GBI score (OR: 8.75 , 95\%CI: 2.07$36.93, p<0.05$ ). Additionally, the clinically significant improvement of the NOSE score was statistically significantly associated with a higher likelihood of clinically significant improvement of the QOD (OR: 3.51,95\%CI: 1.02-12.04, $p<0.05$ ) and SASTscores
(OR: 3.82, 95\%CI: $1.16-12.51, p<0.05$ ) and higher incidence of positive GBI score (OR: $14,95 \% \mathrm{CI}$ : 3.32-58.95, $p<0.001-$ -Table 6). Furthermore, the presence of olfactory dysfunction was an independent predictive factor significantly associated with a lower likelihood of clinically significant improvement BDI score (OR: 0.56, 95\%CI: 0.13-0.76, $p<0.05$ ).

\section{Discussion}

Nasal septal deviation is a common nasal disease causing nasal obstruction symptoms and decreased olfactory function, which, in turn, affect the QoL of the patients. ${ }^{15,46}$ Although there are previous reports on patients' satisfaction and the impact of septoplasty on the QoL of th epatients, ${ }^{23,47-51}$ there are no studies analyzing the impact of olfactory dysfunction on the emotional status and QoL in patients who had septoplasty. The present study assessed olfactory function as a potential predictor of patients' QoL and psychological status improvement, analyzing the correlations of demographic and clinical characteristics of the patients who experience a clinically significant improvement on the emotional status and QoL. The clinical importance of investigating the emotional status and QoL in this group of patients is related to the fact that nasal septal deviation is a common cause of olfactory impairment and nasal obstruction. Psychological status and QoL are likely to be affected due to the chronicity of symptoms, and it is a surgically treatable condition whose outcome measurement is essential to plan personalized patient care. Interesting findings regarding the correlations between patients' demographic and clinical characteristics and QoL, stress levels, depressive mood, and personal benefit from septoplasty were noted.

In the present study as well as in previous researches, ${ }^{52}$ it was observed that patients with septal deviation had impaired olfactory function (lower OT, OD, OI and TDI scores), more severe nasal obstruction symptoms and reduced nasal-symptom related QoL than healthy controls. Furthermore, patients with olfactory deficits due to septal deviation had reduced nasal-symptom related and olfaction-associated QoL compared with normosmic patients and healthy controls. Normosmic patients had less impact on olfaction-associated QoL than patients with impaired olfactory function compared with healthy controls. Six months after septoplasty, probably due 
Table 4 Clinically significant improvement of questionnaire of olfactory disorders and positive change of Glasgow benefit inventory in relation to the demographic and clinical characteristics of the patients

\begin{tabular}{|c|c|c|c|c|c|c|}
\hline & $\begin{array}{l}\text { Clinically } \\
\text { improved } \\
\text { QOD (n, \%) }\end{array}$ & OR $(95 \% \mathrm{CI})$ & $p$-value & $\begin{array}{l}\text { Positive } \\
\text { change } \\
\text { GBI }(n, \%)\end{array}$ & OR $(95 \% \mathrm{CI})$ & $p$-value \\
\hline Age & & & 0.39 & & & 0.55 \\
\hline$\leq 30$ years & $9(30 \%)$ & Ref. & & $15(50 \%)$ & Ref. & \\
\hline$>30$ years & $13(43.3 \%)$ & $1.02(0.97-1.06)$ & & $17(56.7 \%)$ & $1.01(0.97-1.05)$ & \\
\hline Sex & & & 0.429 & & & 0.944 \\
\hline Males & $12(35.2 \%)$ & Ref. & & $18(52.9 \%)$ & Ref. & \\
\hline Females & $10(38.4 \%)$ & $1.53(0.53-4.42)$ & & $14(53.8 \%)$ & $1.03(0.37-2.88)$ & \\
\hline Smoking & & & 0.85 & & & 0.855 \\
\hline No & $15(37.5 \%)$ & Ref. & & $22(55 \%)$ & Ref. & \\
\hline Yes & $7(35 \%)$ & $0.46(0.36-1.21)$ & & $10(50 \%)$ & $0.9(0.3-2.65)$ & \\
\hline Socio-economic status & & & 0.285 & & & 0.804 \\
\hline Low & $4(28.5 \%)$ & Ref. & & $7(50 \%)$ & Ref. & \\
\hline Medium & $8(33.3 \%)$ & $1.26(0.56-2.07)$ & & $13(54.1 \%)$ & $1.02(0.66-2.25)$ & \\
\hline High & $10(45.4 \%)$ & $1.47(0.72-2.98)$ & & $12(54.5 \%)$ & $1.08(0.56-2.11)$ & \\
\hline Clinically improved NOSE & & & 0.009 & & & $<0.001$ \\
\hline No & $9(20 \%)$ & Ref. & & $4(9 \%)$ & Ref. & \\
\hline Yes & $13(63.3 \%)$ & $4.57(1.45-14.38)$ & & $28(88 \%)$ & $11(3.29-36.75)$ & \\
\hline Clinically improved SNOT-22 & & & 0.022 & & & 0.001 \\
\hline No & 7 (22.5\%) & Ref. & & $10(26.2 \%)$ & Ref. & \\
\hline Yes & $15(51.7 \%)$ & $3.67(1.2-11.18)$ & & $22(81.3 \%)$ & $6.6(2.12-20.55)$ & \\
\hline $\begin{array}{l}\text { Olfactory dysfunction } \\
\text { postoperatively }\end{array}$ & & & 0.024 & & & 0.302 \\
\hline No & $17(46.3 \%)$ & Ref. & & $22(53.6 \%)$ & Ref. & \\
\hline Yes & $5(26.3 \%)$ & $0.27(0.18-0.96)$ & & $10(52.6 \%)$ & $0.97(0.6-2.29)$ & \\
\hline
\end{tabular}

Abbreviations: $\mathrm{Cl}$, confidence intervals; GBI, Glasgow benefit inventory; NOSE, nasal obstruction symptom evaluation; OR, odds ratio; $p$-values, univariate linear regression; QOD, questionnaire of olfactory disorders; SNOT 22, SinoNasal outcome test 22.

Data are expressed as number of patients ( $\mathrm{n}$ ) and percentages (\%).

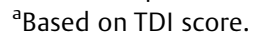

to increased intranasal airflow, an improvement of olfactory function was observed and this was expressed by a significant improvement of TDI scores and all separate olfactory (OT, OD, OI) scores. Previous reports demonstrated similar results. $^{1,11,13-15,46,53}$ Postoperatively, olfaction-associated QoL did not differ significantly between the three groups. Olfaction-related QoL was improved for both normosmic patients and those with olfactory dysfunction. Specifically, the mean value of the QOD score for the group of patients with olfactory deficits postoperatively reached the preoperative mean value of the normosmic patients. The QOD is a symptom-specific (olfaction) QoL measure but is possibly also affected by other nasal symptoms. The effects of other nasal symptoms might have a more prominent negative impact on QoL of patients with olfactory dysfunction because nasal symptoms coexist with impairment in areas such as food enjoyment, harm avoidance, and socializing. ${ }^{2}$ According to the results of the present study, septoplasty leads to olfaction recovery and increase in the number of patients with normal olfactory function. Additionally, it contributes to nasal-symp- tom related and olfaction-associated QoL improvement in patients with olfactory dysfunction and septal deviation, through nasal symptoms resolution and improvement of olfactory status.

Another important finding of the present study was that although in univariate analysis, the presence of postoperative olfactory dysfunction was significantly negatively correlated with clinically significant improvement of QOD, this association became non-significant in multivariate analysis. After screening of all potential demographic and clinical predictors, it was found that clinically significant improvement of nasal obstruction symptom severity (NOSE scores) and nasal-symptom related QoL (SNOT-22 scores) were the only parameters highly associated with an increased likelihood of clinically significant improvement of olfaction-associated QoL (QOD scores). These results support the opinion that there seems to be a disconnection between quantitative assessment of olfactory function and subjective experience of olfactory impairment and that olfactory-specific QoL is affected by additional factors beyond olfactory status. 
Table 5 Clinically significant improvement of short anxiety screening test and Beck depression inventory in relation to the demographic and clinical characteristics of the patients

\begin{tabular}{|l|l|l|l|l|l|l|}
\hline & $\begin{array}{l}\text { Clinically } \\
\text { improved } \\
\text { SAST (n, \%) }\end{array}$ & OR (95\% CI) & $p$-value & $\begin{array}{l}\text { Clinically } \\
\text { improved } \\
\text { BDI (n, \%) }\end{array}$ & OR (95\% CI) & $p$-value \\
\hline Age & & & 0.143 & & & 0.114 \\
\hline$\leq 30$ years & $17(56.7 \%)$ & Ref. & & $10(33.3 \%)$ & Ref. & \\
\hline$>30$ years & $15(50 \%)$ & $0.33(0.18-1.08)$ & & $8(26.7 \%)$ & $0.87(0.69-1.08)$ & \\
\hline Gender & & & 0.944 & & & 0.309 \\
\hline Male & $17(50 \%)$ & Ref. & & $11(32.3 \%)$ & Ref. & \\
\hline Female & $15(58 \%)$ & $1.76(0.34-2.68)$ & & $7(27 \%)$ & $0.55(0.17-1.74)$ & \\
\hline Smoking & & & 0.204 & & & 0.238 \\
\hline No & $20(50 \%)$ & Ref. & & $7(35 \%)$ & Ref. & \\
\hline Yes & $12(45 \%)$ & $0.25(0.17-2.32)$ & & $11(27.5 \%)$ & $0.24(0.17-4.7)$ & \\
\hline Socioeconomic status & & & 0.443 & & & 0.825 \\
\hline Low & $7(50 \%)$ & Ref. & & $5(21.7 \%)$ & Ref. & \\
\hline Medium & $13(54.1 \%)$ & $1.02(0.43-2.12)$ & & $7(25.1 \%)$ & $1.03(0.24-1.98)$ & \\
\hline High & $12(54.5 \%)$ & $1.3(0.66-2.54)$ & & $6(29.2 \%)$ & $1.08(0.52-2.24)$ & \\
\hline Clinically improved NOSE & & & & & 0.029 \\
\hline No & $7(23.3 \%)$ & Ref. & & $6(24 \%)$ & Ref. & \\
\hline Yes & $25(83.3 \%)$ & $4.03(1.37-11.83)$ & & $12(68 \%)$ & $3.82(1.15-12.71)$ & \\
\hline Clinically improved SNOT-22 & & & 0.001 & & & 0.019 \\
\hline No & $9(15 \%)$ & Ref. & & $5(21.3 \%)$ & Ref. & \\
\hline Yes & $23(79.3 \%)$ & $6.39(2.07-19.68)$ & & $13(72.4 \%)$ & $4.22(1.26-14.09)$ & \\
\hline $\begin{array}{l}\text { Olfactory dysfunction } \\
\text { postoperatively }\end{array}$ & & & 0.941 & & & 0.012 \\
\hline No & $22(53.6 \%)$ & Ref. & & $12(65.7 \%)$ & Ref. & \\
\hline Yes & $10(52.6 \%)$ & $0.94(0.35-3.1)$ & & $6(17.2 \%)$ & $0.28(0.14-0.68)$ & \\
\hline
\end{tabular}

Abbreviations: BDI, Beck depression inventory; Cl, confidence intervals; NOSE, nasal obstruction symptom evaluation; OR, odds ratio; $p$-values, univariate linear regression; SAST, short anxiety screening test; SNOT 22, SinoNasal outcome test 22.

Data are expressed as number of patients ( $\mathrm{n}$ ) and percentages (\%).

${ }^{a}$ Based on TDI score.

In the present study, it was found that the whole patient group had higher stress levels (SAST values) than healthy individuals. Patients with olfactory deficits had more anxiety symptoms than patients with normal smell and healthy controls. Stress levels were reduced for the whole patient group after nasal septoplasty, in accordance with the results reported by Hong et al. ${ }^{24}$ However, 6 months after surgery, only patients with normal olfactory function had stress levels that presented no significant differences from healthy controls. Furthermore, clinically significant improvement of nasal obstruction symptom severity (NOSE scores) and nasal-symptom related QoL (SNOT-22 scores), but not the presence of normal olfaction, were highly associated with an increased likelihood of clinically significant improvement of anxiety symptoms (SAST scores). Specifically, patients with clinically significant improvement of nasal obstruction symptom severity and nasal-symptom related QoL were 3.82 and 6.14 times more likely to experience clinically significant improvement of anxiety symptoms
(SAST scores), respectively. These results suggest that the levels of stress in patients treated for septal deviation are less affected by olfactory impairment than what has been reported for patients with other sinonasal diseases, such as chronic rhinosinusitis with or without nasal polyposis. ${ }^{54-56}$ Although patients with olfactory deficits present higher stress levels than normosmic patients, nasal obstruction symptoms appear to be the main factor inducing anxiety symptoms in patients with septal deviation. This finding seems reasonable because nasal obstruction can be connected with symptoms such as insomnia/sleep disturbances and headaches, which are assessed with the use of the SAST questionnaire.

Regarding the emotional status, patients with olfactory deficits had more depressive mood (BDI scores) compared with patients with normal olfactory function and healthy controls, both before and 6 months after surgery. Previous studies evaluating the impact of olfactory disorders, due to various causes, on the QoL of patients ${ }^{2,4-6}$ demonstrated that 
Table 6 Multivariate logistic regression analysis between predictor variables and clinically significant improvement of QOD, SAST and BDI scores and positive change of GBI scores

\begin{tabular}{|l|l|l|}
\hline & OR (95\% CI) & $p$-value \\
\hline QOD & & \\
\hline Clinically improved NOSE & $3.51(1.02-12.04)$ & 0.046 \\
\hline Clinically improved SNOT-22 & $3.79(1.11-12.88)$ & 0.033 \\
\hline $\begin{array}{l}\text { Olfactory Dysfunction } \\
\text { postoperatively }\end{array}$ & $0.23(0.16-1.8)$ & 0.204 \\
\hline SAST & & \\
\hline Clinically improved NOSE & $3.82(1.16-12.51)$ & 0.027 \\
\hline Clinically improved SNOT-22 & $6.14(1.87-20.07)$ & 0.003 \\
\hline BDI & & \\
\hline Clinically improved NOSE & $2.67(0.69-10.39)$ & 0.154 \\
\hline Clinically improved SNOT 22 & $5.61(1.35-23.15)$ & 0.017 \\
\hline $\begin{array}{l}\text { Olfactory Dysfunction } \\
\text { postoperatively }\end{array}$ & $0.56(0.13-0.76)$ & 0.021 \\
\hline GBI & & \\
\hline Clinically improved NOSE & $14(3.32-58.95)$ & $<0.001$ \\
\hline Clinically improved SNOT-22 & $8.75(2.07-36.93)$ & 0.003 \\
\hline
\end{tabular}

Abbreviations: BDI, Beck depression inventory; GBI, Glasgow benefit inventory; NOSE, nasal obstruction symptom evaluation; QOD, questionnaire of olfactory disorders; SAST, short anxiety screening test; SNOT-22, SinoNasal outcome test-22.

Data are expressed as odds ratios (OR) with their $95 \%$ confidence intervals $(\mathrm{Cl})$. Only variables with statistical significance during univariate linear regression analysis are included. pvalues: Multivariate logistic regression analysis.

individuals with olfactory dysfunction experience reduced food enjoyment, problems related to personal hygiene (insecurity about personal body odor), reduced participation in social life and limitations with sensing warning signals (gas/smoke). It can be assumed that the aforementioned daily life restrictions may impair QoL and impact the emotional status of patients with olfactory deficits related to septal deviation. The negative impact of olfactory dysfunction on patients' emotional status could also be related to the connection between olfactory system and structures of the limbic system like the amygdala and orbitofrontal cortex, ${ }^{57}$ which mainly contribute to the development of depressive symptoms. ${ }^{58}$ It is noteworthy that, clinically significant improvement of nasal-symptom related QoL (SNOT-22 scores) was associated with an increased likelihood of clinically significant improvement of depressive mood (BDI scores). Patients with clinically significantly improved SNOT-22 scores were 5.61 times more likely to experience clinically significant improvement in levels of depression. In contrast, impaired olfaction postoperatively was correlated with reduced incidence of improved BDI scores. Normosmic patients were 4.37 times more likely to experience clinically significant improvement of depressive mood (BDI scores) than patients with olfactory deficits. These findings indicate that olfactory impairment due to septal deviation appears to have a negative effect on patients' emotional profile.
According to the results of the present study, olfactory impairment had a negative impact on patient-reported benefit from surgery, as patients with impaired smell appeared less satisfied than normosmic patients. However, olfactory status was not found to have a significant impact on the likelihood of personal benefit from septoplasty. Similarly, none of the patients' demographics affected the subjective evaluation of surgical outcome, in agreement with previous studies which examined age, gender, smoking ${ }^{19,21,24,25,48}$ and socio-economic status. ${ }^{19}$ Clinically significant improvement of nasal obstruction and nasalsymptom related QoL were the only predictive factors significantly associated with subjective surgical outcome. Specifically, patients with clinically significant nasal obstruction symptom resolution and nasal-symptom related QoL improvement were 14 and 8.75 times more likely to experience higher personal benefit from surgery, respectively. This finding is in accordance with other reports, which demonstrated that patients with more severe nasal symptoms preoperatively had more potential of improvement of their health status after septoplasty and were more satisfied with surgical outcome. ${ }^{19,21,23,24,49}$ Thus, it seems that personal benefit from septoplasty is mainly affected by nasal symptom resolution rather than improvement of olfactory function.

An interesting finding of the present study was that there were no significant differences between general QoL (SF-36 scores) of healthy controls and patients with septal deviation, either with olfactory deficits or normosmics. Probably, nasal obstruction and olfactory impairment related to septal deviation do not cause a deterioration of health status significant enough to be depicted in this general-health status questionnaire. ${ }^{50,51}$ In contrast, the SNOT-22 is a specific nasal-symptom related QoL questionnaire, and it covers the effects of physical health, functional limitations, and emotional aspects of QoL related to the nasal functions, including olfaction. That explains why, according to the results of the present study, clinically significant improvement of the SNOT-22 score was an independent predictor highly associated with clinically significant of olfaction-associated QoL, psychological status (anxiety symptoms and depressive mood), and a higher likelihood of patients-reported benefit from the surgical procedure.

To the best of our knowledge, this is the first study to address the association of olfactory function with clinically significant improvement of QoL and psychological outcomes of patients who undergo septoplasty. In the present study, validated tools were used to measure general, disease-specific and olfaction-specific QoL, emotional status, and patient outcome satisfaction and comparisons with healthy controls were performed. Additionally, quantitative smell tests for the measurement of olfactory performance were utilized, providing clinically important data for the olfactory function in patients treated with septoplasty. Future studies may provide useful knowledge on the clinical importance of olfactory testing prior to surgery for appropriate consultation of patients. 


\section{Conclusion}

Nasal septoplasty leads to better olfactory function along with nasal obstruction resolution and improvement of nasal symptom-related QOL. Olfactory impairment related to septal deviation has a negative impact on psychological status and disease-specific QoL. Although improvement of nasal obstruction and nasal-symptom related QoL were the only factors significantly associated with patients' evaluation of surgical outcome, patients with olfactory deficits were less satisfied with the surgical procedure than normosmics and experienced greater stress and depressive mood. Quantitative evaluation of the olfactory status of patients treated for septal deviation is important for optimal assessment of the patients and consultation regarding the anticipated septoplasty outcomes.

\section{Conflict of Interests}

The authors have no conflict of interests to declare.

\section{References}

1 Damm M, Eckel HE, Jungehülsing M, Hummel T. Olfactory changes at threshold and suprathreshold levels following septoplasty with partial inferior turbinectomy. Ann Otol Rhinol Laryngol 2003;112 (01):91-97

2 Croy I, Nordin S, Hummel T. Olfactory disorders and quality of life-an updated review. Chem Senses 2014;39(03):185-194

3 Marschner H, Gudziol H, Guntinas-Lichius O. [Olfactory dysfunctions are substantially more frequent than they are complained]. Laryngorhinootologie 2010;89(11):654-659

4 Shu CH, Lee PO, Lan MY, Lee YL. Factors affecting the impact of olfactory loss on the quality of life and emotional coping ability. Rhinology 2011;49(03):337-341

5 Smeets MAM, Veldhuizen MG, Galle S, et al. Sense of smell disorder and health-related quality of life. Rehabil Psychol 2009;54(04):404-412

6 Brämerson A, Nordin S, Bende M. Clinical experience with patients with olfactory complaints, and their quality of life. Acta Otolaryngol 2007;127(02):167-174

7 Moore M, Eccles R. Objective evidence for the efficacy of surgical management of the deviated septum as a treatment for chronic nasal obstruction: a systematic review. Clin Otolaryngol 2011;36 (02):106-113

8 de $\mathrm{Ru}$ JA. Septoplasty is a proven and effective procedure: an expert's view of a burning issue. B-ENT 2015;11(04):257-262

9 Valsamidis K, Titelis K, Rachovitsas D, Konstantinidis I, Markou K, Triaridis S. Long-Term Evaluation of Nasal Septoplasty Followed by Inferior Turbinate Cauterization for the Treatment of Nasal Obstruction using Objective and Subjective Methods. Int Arch Otorhinolaryngol 2018;22(03):284-290

10 Tsang CLN, Nguyen T, Sivesind T, Cervin A. Long-term patientrelated outcome measures of septoplasty: a systematic review. Eur Arch Otorhinolaryngol 2018;275(05):1039-1048

11 Aydoğdu I, Atar Y, Aydoğdu Z, et al. Comparison of Olfactory Function and Quality of Life With Different Surgical Techniques for Nasal Septum Deviation. J Craniofac Surg 2019;30(02): 433-436

12 Haytoğlu S, Dengiz R, Muluk NB, Kuran G, Arikan OK. Effects of septoplasty on olfactory function evaluated by the Brief Smell Identification Test: A study of 116 patients. Ear Nose Throat J 2017;96(10-11):433-438

13 Türk B, Akpinar M, Altundağ A, Kirik MÖ, Ünsal Ö, Coşkun BU. The Effect of External Approach Septoplasty on Olfactory Function. J Craniofac Surg 2017;28(07):1675-1678
14 Kilicaslan A, Acar GO, Tekin M, Ozdamar OI. Assessment the longterm effects of septoplasty surgery on olfactory function. Acta Otolaryngol 2016;136(10):1079-1084

15 Gupta N, Singh PP, Bagla RK. Will septal correction surgery for deviated nasal septum improve the sense of smell? A prospective study. Surg Res Pract 2015;2015:496542. Doi: 10.1155/2015/ 496542

16 Fyrmpas G, Tsalighopoulos M, Constantinidis J. Lateralized olfactory difference in patients with a nasal septal deviation before and after septoplasty. Hippokratia 2012;16(02):166-169

17 Pade J, Hummel T. Olfactory function following nasal surgery. Laryngoscope 2008;118(07):1260-1264

18 Pfaar O, Hüttenbrink KB, Hummel T. Assessment of olfactory function after septoplasty: a longitudinal study. Rhinology 2004;42(04): 195-199

19 Valsamidis K, Titelis K, Karkos P, Markou K, Constantinidis J, Triaridis S. Predictive factors of patients' general quality of life after nasal septoplasty. Eur Arch Otorhinolaryngol 2019;276(02):429-438

20 Cantone E, Ricciardiello F, Oliva F, De Corso E, Iengo M. Septoplasty: is it possible to identify potential "predictors" of surgical success? Acta Otorhinolaryngol Ital 2018;38(06):528-535

21 Stewart MG, Smith TL, Weaver EM, et al. Outcomes after nasal septoplasty: results from the Nasal Obstruction Septoplasty Effectiveness (NOSE) study. Otolaryngol Head Neck Surg 2004; 130(03):283-290

22 Karatzanis AD, Fragiadakis G, Moshandrea J, Zenk J, Iro H, Velegrakis GA. Septoplasty outcome in patients with and without allergic rhinitis. Rhinology 2009;47(04):444-449

23 Hytönen ML, Lilja M, Mäkitie AA, Sintonen H, Roine RP. Does septoplasty enhance the quality of life in patients? Eur Arch Otorhinolaryngol 2012;269(12):2497-2503

24 Hong SD, Lee NJ, Cho HJ, et al. Predictive factors of subjective outcomes after septoplasty with and without turbinoplasty: can individual perceptual differences of the air passage be a main factor? Int Forum Allergy Rhinol 2015;5(07):616-621

25 Shiryaeva O, Tarangen M, Gay C, Døsen LK, Haye R. Preoperative signs and symptoms as prognostic markers in nasal septoplasty. Int J Otolaryngol 2017;2017:4718108. Doi: 10.1155/2017/47181

26 Kobal G, Hummel T, Sekinger B, Barz S, Roscher S, Wolf S. 'Sniffin' sticks": screening of olfactory performance. Rhinology 1996;34 (04):222-226

27 Hummel T, Sekinger B, Wolf SR, Pauli E, Kobal G. 'Sniffin' sticks': olfactory performance assessed by the combined testing of odor identification, odor discrimination and olfactory threshold. Chem Senses 1997;22(01):39-52

28 Oleszkiewicz A, Schriever VA, Croy I, Hähner A, Hummel T. Updated Sniffin' Sticks normative data based on an extended sample of 9139 subjects. Eur Arch Otorhinolaryngol 2019;276(03):719-728

29 Konstantinidis I, Printza A, Genetzaki S, Mamali K, Kekes G, Constantinidis J. Cultural adaptation of an olfactory identification test: the Greek version of Sniffin' Sticks. Rhinology 2008;46(04): 292-296

30 Klimek L, Hummel T, Moll B, Kobal G, Mann WJ. Lateralized and bilateral olfactory function in patients with chronic sinusitis compared with healthy control subjects. Laryngoscope 1998; 108(1 Pt 1):111-114

31 Stewart MG, Witsell DL, Smith TL, Weaver EM, Yueh B, Hannley MT. Development and validation of the Nasal Obstruction Symptom Evaluation (NOSE) scale. Otolaryngol Head Neck Surg 2004; 130(02):157-163

32 Lachanas VA, Tsiouvaka S, Tsea M, Hajiioannou JK, Skoulakis CE. Validation of the nasal obstruction symptom evaluation (NOSE) scale for Greek patients. Otolaryngol Head Neck Surg 2014;151 (05):819-823

33 Browne JP, Hopkins C, Slack R, Cano SJ. The Sino-Nasal Outcome Test (SNOT): can we make it more clinically meaningful? Otolaryngol Head Neck Surg 2007;136(05):736-741 
34 Lachanas VA, Tsea M, Tsiouvaka S, Hajiioannou JK, Skoulakis CE, Bizakis JG. The sino-nasal outcome test (SNOT)-22: validation for Greek patients. Eur Arch Otorhinolaryngol 2014;271(10):2723-2728

35 Frasnelli J, Hummel T. Olfactory dysfunction and daily life. Eur Arch Otorhinolaryngol 2005;262(03):231-235

36 Simopoulos E, Katotomichelakis M, Gouveris H, Tripsianis G, Livaditis M, Danielides V. Olfaction-associated quality of life in chronic rhinosinusitis: adaptation and validation of an olfaction-specific questionnaire. Laryngoscope 2012;122(07):1450-1454

37 Grammatikopoulos IA, Sinoff G, Alegakis A, Kounalakis D, Antonopoulou M, Lionis C. The Short Anxiety Screening Test in Greek: translation and validation. Ann Gen Psychiatry 2010;9(01):1

38 Sinoff G, Ore L, Zlotogorsky D, Tamir A. Short Anxiety Screening Test-a brief instrument for detecting anxiety in the elderly. Int J Geriatr Psychiatry 1999;14(12):1062-1071

39 Beck AT, Steer RA, Brown GK. Manual for the Beck Depression Inventory. San Antonio, TX: Psychological Corporation; 1996

40 Giannakou M, Roussi P, Kosmides ME, Kiosseoglou G. Adaptation of the beck depression inventory-II to greek population. Hell J Psychol 2013;10(02):120-146

41 Ware JE Jr, Gandek B. Qverview of the SF-36 Health Survey and the international quality of life assessment (IQOLA) Project. J Clin Epidemiol 1998;51(11):903-912

42 Anagnostopoulos F, Niakas D, Pappa E. Construct validation of the Greek SF-36 Health Survey. Qual Life Res 2005;14(08):1959-1965

43 Robinson K, Gatehouse S, Browning GG. Measuring patient benefit from otorhinolaryngological surgery and therapy. Ann Otol Rhinol Laryngol 1996;105(06):415-422

44 Konstantinidis I, Triaridis S, Printza A, Triaridis A, Noussios G, Karagiannidis K. Assessment of patient benefit from septo-rhinoplasty with the use of Glasgow Benefit Inventory (GBI) and Nasal Symptom Questionnaire (NSQ). Acta Otorhinolaryngol Belg 2003; 57(02):123-129

45 Norman GR, Sloan JA, Wyrwich KW. Interpretation of changes in health-related quality of life: the remarkable universality of half a standard deviation. Med Care 2003;41(05):582-592

46 Berkiten G, Kumral TL, Saltürk Z, et al. Effect of Deviated Nasal Septum Type on Nasal Mucociliary Clearance, Olfactory Function,
Quality of Life, and Efficiency of Nasal Surgery. J Craniofac Surg 2016;27(05):1151-1155

47 Konstantinidis I, Triaridis S, Triaridis A, Karagiannidis K, Kontzoglou G. Long term results following nasal septal surgery. Focus on patients' satisfaction. Auris Nasus Larynx 2005;32(04): 369-374

48 Mondina M, Marro M, Maurice S, Stoll D, de Gabory L. Assessment of nasal septoplasty using NOSE and RhinoQoL questionnaires. Eur Arch Otorhinolaryngol 2012;269(10):2189-2195

49 Uppal S, Mistry H, Nadig S, Back G, Coatesworth A. Evaluation of patient benefit from nasal septal surgery for nasal obstruction. Auris Nasus Larynx 2005;32(02):129-137

50 Calder NJ, Swan IRC. Outcomes of septal surgery. J Laryngol Otol 2007;121(11):1060-1063

51 Arunachalam PS, Kitcher E, Gray J, Wilson JA. Nasal septal surgery: evaluation of symptomatic and general health outcomes. Clin Otolaryngol Allied Sci 2001;26(05):367-370

52 Bugten V, Nilsen AH, Thorstensen WM, Moxness MH, Amundsen MF, Nordgård S. Quality of life and symptoms before and after nasal septoplasty compared with healthy individuals. BMC Ear Nose Throat Disord 2016;16:13

53 Choi YS, Ryu YJ, Rhee J, et al. Clinical Implications of Septal Deviation in Lateralized Olfaction. Clin Exp Otorhinolaryngol 2016;9(01):39-43

54 Katotomichelakis M, Simopoulos E, Tripsianis G, et al. Predictors of quality of life outcomes in chronic rhinosinusitis after sinus surgery. Eur Arch Otorhinolaryngol 2014;271(04):733-741

55 Katotomichelakis M, Simopoulos E, Tripsianis G, et al. Improvement of olfactory function for quality of life recovery. Laryngoscope 2013;123(11):E10-E16

56 Mattos JL, Schlosser RJ, Storck KA, Soler ZM. Understanding the relationship between olfactory-specific quality of life, objective olfactory loss, and patient factors in chronic rhinosinusitis. Int Forum Allergy Rhinol 2017;7(07):734-740

57 Gottfried JA. Smell: central nervous processing. Adv Otorhinolaryngol 2006;63:44-69

58 Palazidou E. The neurobiology of depression. Br Med Bull 2012; 101:127-145 